\title{
Role of the Radiotherapy Boost on Local Control in Ductal Carcinoma In Situ
}

\author{
Olivier Riou,, ${ }^{1}$ Claire Lemanski, ${ }^{1}$ Vanessa Guillaumon, ${ }^{2}$ Olivier Lauche, ${ }^{1}$ \\ Pascal Fenoglietto, ${ }^{1}$ Jean-Bernard Dubois, ${ }^{1}$ and David Azria ${ }^{1}$ \\ ${ }^{1}$ Département d'Oncologie Radiothérapie, CRLC Val d'Aurelle-Paul Lamarque, 34298 Montpellier, France \\ ${ }^{2}$ Département de Recherche Clinique, CRLC Val d'Aurelle-Paul Lamarque, 34298 Montpellier, France
}

Correspondence should be addressed to Olivier Riou, riouo@hotmail.com

Received 2 December 2011; Accepted 23 January 2012

Academic Editor: Bruno Cutuli

Copyright (c) 2012 Olivier Riou et al. This is an open access article distributed under the Creative Commons Attribution License, which permits unrestricted use, distribution, and reproduction in any medium, provided the original work is properly cited.

\begin{abstract}
Ductal carcinoma in situ of the breast is associated with low mortality rates, but local relapse is a matter of concern in this disease. Risk factors for local relapse include young age, close or positive margins, and tumor necrosis. Whole breast irradiation following breast-conserving surgery for ductal carcinoma in situ significantly reduces the risk of local relapse as compared to breastconserving surgery alone. Studies point to similar outcomes between breast-conserving surgery plus radiotherapy and mastectomy, in the absence of extensive disease. A complementary boost to the surgical bed improves outcomes for patients with invasive breast cancer. However, the effect of this strategy has never been prospectively reported for ductal carcinoma in situ. Two randomized controlled trials assessing this issue are ongoing. This paper represents an update on available literature about radiotherapy for DCIS with a special focus on the role of a radiotherapy boost to the tumor bed.
\end{abstract}

\section{Introduction}

Ductal carcinoma in situ (DCIS) is a proliferation of malignant cells inside galactophoric ducts without basal membrane invasion. Its incidence has dramatically increased in the recent years due to the widespread use of mammographic screening. Accounting for approximately 20 to $30 \%$ of the breast cancer cases [1], DCIS is heterogeneous in clinical presentation, varying from a palpable mass, mammographically detected tumor, or nipple discharge [2]. Despite a high cure rate, invasive recurrence and death may occur in case of insufficient local treatment. Patients with clinically large, multicentric, and extensive tumors are more likely to undergo mastectomy than breast-conserving surgery (BCS), because of a higher risk of recurrence [3]. Likewise, mastectomy may be the preferred strategy in case of diffuse suspicious-appearing microcalcifications in the breast, inability to obtain margin control by lumpectomy and/or reexcision(s), medical contraindication to irradiation, and when an unfavorable tumor-to-breast size ratio does not permit margin-negative lumpectomy with cosmetically acceptable results [4].

No randomized controlled trials comparing mastectomy with more conserving management are available, but different studies point to similar outcomes between BCS plus radiotherapy and mastectomy, whereas BCS alone tends to be inferior [5]. Therefore, BCS plus radiotherapy is an accepted strategy when mastectomy can be avoided, in view of the morbidity of radical surgery and the favorable prognosis of such patients. A number of randomized controlled trials of adjuvant radiotherapy have indeed demonstrated a reduced risk of both invasive and local recurrences, as well as a low risk of side effects $[6,7]$.

A radiation boost to the tumor bed has been shown to significantly improve local control in patients with invasive breast cancer $[8,9]$. However, the usefulness of a boost has not been so well assessed in the setting of DCIS, and prospective studies are missing.

This paper aims at updating available literature on the subject and at developing the rationale for randomized 
multicenter phase 3 studies assessing the role of surgical bed boost following whole breast irradiation (WBI).

\section{Risk Factors for Local Relapse following Breast-Conserving Therapy}

Several studies have attempted to identify the local recurrence risk factors following breast-conserving surgery [10]. Clinical factors have been determined by uni- and multivariate analysis of randomized trials and multicenter retrospective studies. These findings suggest that a family history of breast cancer, a young-onset disease, and a palpable tumor of more than $1 \mathrm{~cm}$ are factors that may adversely affect local control [11-13]. Moreover, the comedocarcinoma subtype, a histopathology size greater than $10 \mathrm{~mm}$, necrosis, and positive margins have been shown to be statistically significant predictive factors for recurrence in women under 40 years old [14].

Close or positive margins are considered to be risk factors for recurrence, whether patients are irradiated or not. In the B-24 and EORTC 10853 trials, although patients were supposed to have free margins for inclusion $[15,16]$, a central pathology review of the specimens found a significant amount of positive and unknown margins [17]. In these trials, positive margins were found to be independent factors for the development of local relapse after BCS [18]. According to the meta-analysis of Dunne et al., a margin threshold of $2 \mathrm{~mm}$ may be sufficient when BCS is combined with RT [19].

Apart from margins status, tumor necrosis appears to be an important relapse risk factor, even in multifactorial analysis [20]. The histological size, the Scarff-Bloom-Richardson grade, and the degree of differentiation are minor prognostic factors. No prognostic biological or genetic factors have been individualized so far in ductal carcinoma in situ [21].

\section{The Influence of Adjuvant Radiotherapy in the Conserving Treatment of DCIS}

The role of WBI is to reduce the rate of local relapse and to allow for breast conservation. Monocenter and multicenter retrospective studies with 5 to 15 years of followup found recurrence rates of about 7 to $17 \%$. About half of recurrences were invasive, and the metastatic rate was less than $10 \%$. Nevertheless, these studies had heterogeneous inclusion criteria, surgical treatment, radiation dose, and followup [22].

Four randomized controlled trials have assessed the role of irradiation in this setting. A worldwide collaboration group, the Early Breast Cancer Trialists' Collaborative Group (EBCTCG), has centrally reviewed these four trials to give an updated overview of the impact of radiotherapy in DCIS. The latest report confirmed a $15.2 \%$ reduction in the absolute 10 -year risk of ipsilateral breast event, regardless of age at diagnosis, extent of breast-conserving therapy, use of tamoxifen, margin status, comedonecrosis, focality, grade, or tumor size. The amount of risk reduction was higher in older women ( $>50$ years). However, no significant effect on over- all mortality or breast cancer mortality was found after a followup of 10 years [7].

3.1. NSABP B17. The NSABP B17 trial was undertaken in the United States and Canada from 1985 to 1990. The results of this trial were first published in 1993 and then updated in 2001 [23, 24]. A total of 818 patients were randomized, after initial lumpectomy for DCIS, to either surveillance or WBI to 50 Gy in 25 fractions without tumor bed boost. After a mean followup of 129 months, a 57\% reduction in the risk of invasive and in situ breast recurrences was seen. No differences in overall survival, metastatic, or contralateral cancer rates were shown. A pooled analysis of this study and of the NSABP B24 trial confirmed a 52\% reduction in ipsilateral breast recurrence risk at 15 years when patients were offered radiation therapy [25].

3.2. EORTC 10853. This trial was conducted in Europe from 1986 to 1996 and accrued one thousand and ten DCIS patients with free margins after lumpectomy. First analysis after a followup of 4.2 years showed a significant reduction in invasive relapse risk, an increased risk of contralateral cancer, and equivalent survival and metastatic rates [17]. An update after a followup of 10.5 years confirmed these results, with a $48 \%$ reduction in intraductal recurrences and a $42 \%$ decrease in invasive relapses [18].

3.3. UK/ANZ DCIS Trial. This English study was a multicenter randomized trial which assessed both the influence of WBI and the role of tamoxifen adjuvant treatment following BCS. Randomization was independent for radiotherapy and tamoxifen, stratified by screening center, and blocked in groups of four [26]. One thousand seven-hundred and one patients were accrued and one thousand and thirty patients were randomized to radiotherapy or observation. This trial has been recently updated after a followup of 12.7 years [27]. The overall reduction in local relapse was 59\%, and the ipsilateral invasive disease risk reduction was $68 \%$. No difference in the number of contralateral cancer cases was seen. The risk reduction was similar whether patients had tamoxifen or not.

3.4. SweDCIS Trial. This multicenter Swedish trial enrolled DCIS patients treated by BCS with tumor-free margins. One thousand and forty-six women were randomized to either radiotherapy or no radiotherapy. Once again, the analysis after a followup of 5.2 years showed a 3 -fold reduction in invasive and in situ local relapses [28]. An updated analysis published in 2008 confirmed a relative risk reduction of $60 \%$ in local recurrence [29]. Women more than 50 years seemed to benefit most from adjuvant radiotherapy in this trial, and the authors concluded that older age should not preclude DCIS women from radiotherapy.

\section{The Role of the Radiotherapy Boost following BCS and WBI in DCIS}

The rationale for dose escalation to the tumor bed relies on the frequent presence of residual tumor cells in a $10 \mathrm{~mm}$ 
radius of the tumor. A dose of $50 \mathrm{~Gy}$ does not seem to be high enough to kill such remaining cells. However, wider excisions with extensive margins significantly alter the cosmetic results. Margins greater than 2 or $3 \mathrm{~mm}$ are considered safe, despite a risk of remaining tumor cells of up to $20 \%$ $[19,22]$. This risk legitimates dose escalation studies in DCIS.

Several retrospective studies have tried to assess the role of a boost following WBI for DCIS.

An international multicenter retrospective study was performed on 373 patients, aged 45 years or less, treated in 18 institutions [30]. Forty five percent of them underwent WBI, $40 \%$ underwent WBI plus a boost, and $15 \%$ had no radiotherapy. The relapse-free survival rates at ten years were $46 \%$ without radiotherapy, $72 \%$ in the WBI group, and $86 \%$ in the WBI plus boost group. Differences were statistically significant with an overall risk reduction for local relapse of $66 \%$ with WBI and $85 \%$ with WBI and boost. In multivariate analysis, the margins status and the radiation dose were the only two independent factors for relapse-free survival. No difference in overall survival was found.

Another study by Wong et al. confirmed the favorable effect of the radiation boost, with no local relapse observed among 79 patients receiving a boost, whereas 8 of 141 patients in the "no-boost" group experienced in-breast local recurrence [31]. These results were obtained despite a higher risk for local relapse in the boost group: $48 \%$ (boost group) versus $8 \%$ (no boost group) had positive or less than $1 \mathrm{~mm}$ margins.

On the contrary, other reports found no difference in local relapse according to the total radiation dose level: $<60$ Gy versus $>60$ Gy [32], <60 Gy versus 60-66 Gy versus $>66 \mathrm{~Gy}[13,33,34]$. In the study by Wai et al., only $50 \%$ of patients had radiotherapy after BCS, including $35 \%$ receiving WBI without boost and 15\% receiving WBI plus a boost to the lumpectomy bed [35]. Moreover, partial breast boost was used primarily in subjects with positive or close margins, which could alter the outcome of these patients and explain these different results. If a retrospective cohort study of 208 DCIS patients with close or focally involved margins after BCS appeared to show a similar effect of a 16-Gy boost to that of a reexcision [36], prospective studies are warranted to confirm this finding before reexcision may be avoided in all cases.

\section{Ongoing Clinical Trials and Future Directions}

Two randomized controlled trials addressing the role of a 16 Gy boost are ongoing, and patients are currently being recruited.

An international trial of the Trans-Tasman Radiation Oncology Group, started in 2008, simultaneously evaluates the role of the boost to the tumor bed and the effect of hypofractionation on outcomes in DCIS. Patients are divided into 3 strata and randomized within those groups: group A is designed to evaluate the effect of the boost and of the fractionation schedule, group B, the boost after 50 Gy in 25 fractions of WBI, and group C assesses the interest of the boost after $42.5 \mathrm{~Gy}$ in 16 fractions and 22 days of WBI.
In the French multicenter prospective randomized Bonbis trial, DCIS women aged 18 and over are randomly assigned to a 16 Gy boost or no boost, following BCS and the delivery of $50 \mathrm{~Gy}$ in 25 fractions to the whole breast [37]. Nearly half of the patients have been enrolled so far (900 of the planned 1950 women). Inclusion criteria include tumor-free margins, the presence of surgical clips in the lumpectomy bed to ease the boost delivery, and no past history of cancer. A centralized review of all specimens is performed, as well as an assessment of dummy runs and technical radiotherapy cases. Radiotherapy should start within 12 weeks of surgery. Translational studies investigating candidate genes and a predictive test of late toxicity will hopefully help to individualize patients who can be safely treated with dose escalation to the tumor bed.

\section{Summary}

BCS plus WBI is an accepted strategy for DCIS when mastectomy can be avoided. The effect of a complementary boost to the tumor bed has never been prospectively assessed. Two ongoing randomized controlled trials addressing this issue should help to individualize patients who may benefit from this treatment.

\section{Conflict of Interests}

The authors declare that they have no conflict of interests.

\section{References}

[1] B. A. Virnig, T. M. Tuttle, T. Shamliyan, and R. L. Kane, "Ductal carcinoma in Situ of the breast: a systematic review of incidence, treatment, and outcomes," Journal of the National Cancer Institute, vol. 102, no. 3, pp. 170-178, 2010.

[2] K. Mokbel and B. Cutuli, "Heterogeneity of ductal carcinoma in situ and its effects on management," Lancet Oncology, vol. 7, no. 9, pp. 756-765, 2006.

[3] I. Barillot, B. Cutuli, and L. Arnould, "Ductal in situ carcinoma: is it ethical to consider the breast conserving therapy as a standard?" Cancer/Radiotherapie, vol. 8, no. 1, pp. 9-20, 2004.

[4] L. A. Newman, "Local control of ductal carcinoma in situ based on tumor and patient characteristics: the surgeon's perspective," Journal of the National Cancer Institute. Monographs, no. 41, pp. 152-157, 2010.

[5] R. L. Kane, B. A. Virnig, T. Shamliyan, S.-Y. Wang, T. M. Tuttle, and T. J. Wilt, "The impact of surgery, radiation, and systemic treatment on outcomes in patients with ductal carcinoma in situ," Journal of the National Cancer Institute. Monographs, no. 41, pp. 130-133, 2010.

[6] N. Bijker and G. van tienhoven, "Local and systemic outcomes in DCIS based on tumor and patient characteristics: the radiation oncologist's perspective," Journal of the National Cancer Institute, no. 41, pp. 178-180, 2010.

[7] C. Correa, P. McGale, C. Taylor et al., "Overview of the randomized trials of radiotherapy in ductal carcinoma in situ of the breast," Journal of the National Cancer Institute, no. 41, pp. 162-177, 2010.

[8] P. Romestaing, Y. Lehingue, C. Carrie et al., "Role of a 10Gy boost in the conservative treatment of early breast cancer: 
results of a randomized clinical trial in Lyon, France," Journal of Clinical Oncology, vol. 15, no. 3, pp. 963-968, 1997.

[9] H. Bartelink, J.-C. Horiot, P. M. Poortmans et al., "Impact of a higher radiation dose on local control and survival in breastconserving therapy of early breast cancer: 10-year results of the randomized boost versus no boost EORTC 22881-10882 trial," Journal of Clinical Oncology, vol. 25, no. 22, pp. 32593265, 2007.

[10] S. J. Schnitt, "Local outcomes in ductal carcinoma in situ based on patient and tumor characteristics," Journal of the National Cancer Institute, no. 41, pp. 158-161, 2010.

[11] P. Fourneret, X. Artignan, J. De Cornulier et al., "Retrospective analysis of 108 ductal carcinomas in situ of the breast treated by radiosurgery association," Cancer/Radiotherapie, vol. 10, no. 8, pp. 550-558, 2006.

[12] B. Cutuli, C. Cohen-Solal-le Nir, B. De Lafontan et al., "Breastconserving therapy for ductal carcinoma in situ of the breast: the French Cancer Centers' experience," International Journal of Radiation Oncology Biology Physics, vol. 53, no. 4, pp. 868$879,2002$.

[13] L. J. Solin, A. Fourquet, F. A. Vicini et al., "Long-term outcome after breast-conservation treatment with radiation for mammographically detected ductal carcinoma in situ of the breast," Cancer, vol. 103, no. 6, pp. 1137-1146, 2005.

[14] C. Tunon-de-Lara, C. Lemanski, C. Cohen-Solal-Le-Nir et al., "Ductal carcinoma in situ of the breast in younger women: a subgroup of patients at high risk," European Journal of Surgical Oncology, vol. 36, no. 12, pp. 1165-1171, 2010.

[15] B. Fisher, J. Dignam, N. Wolmark et al., "Tamoxifen in treatment of intraductal breast cancer: national surgical adjuvant breast and bowel project B-24 randomised controlled trial," Lancet, vol. 353, no. 9169, pp. 1993-2000, 1999.

[16] J.-P. Julien, N. Bijker, I. S. Fentiman et al., "Radiotherapy in breast-conserving treatment for ductal carcinoma in situ: first results of the EORTC randomised phase III trial 10853," Lancet, vol. 355, no. 9203, pp. 528-533, 2000.

[17] N. Bijker, J. L. Peterse, L. Duchateau et al., "Risk factors for recurrence and metastasis after breast-conserving therapy for ductal carcinoma-in-situ: analysis of European Organization for Research and Treatment of Cancer Trial 10853," Journal of Clinical Oncology, vol. 19, no. 8, pp. 2263-2271, 2001.

[18] N. Bijker, P. Meijnen, J. L. Peterse et al., "Breast-conserving treatment with or without radiotherapy in ductal carcinomain-situ: ten-year results of european organisation for research and treatment of cancer randomized phase III trial 10853a study by the EORTC breast cancer cooperative group and EORTC radiotherapy group," Journal of Clinical Oncology, vol. 24, no. 21, pp. 3381-3387, 2006.

[19] C. Dunne, J. P. Burke, M. Morrow, and M. R. Kell, "Effect of margin status on local recurrence after breast conservation and radiation therapy for ductal carcinoma in situ," Journal of Clinical Oncology, vol. 27, no. 10, pp. 1615-1620, 2009.

[20] A. Ringberg, H. Nordgren, S. Thorstensson et al., "Histopathological risk factors for ipsilateral breast events after breast conserving treatment for ductal carcinoma in situ of the breast - Results from the Swedish randomised trial," European Journal of Cancer, vol. 43, no. 2, pp. 291-298, 2007.

[21] E. R. Fisher, S. R. Land, R. S. Saad et al., "Pathologic variables predictive of breast events in patients with ductal carcinoma in situ," American Journal of Clinical Pathology, vol. 128, no. 1, pp. 86-91, 2007.
[22] B. Cutuli, A. Fourquet, E. Luporsi et al., "Standards, options and recommendations for the management of ductal carcinoma in situ of the breast (DCIS): update 2004," Bulletin du Cancer, vol. 92, no. 2, pp. 155-168, 2005.

[23] B. Fisher, J. Costantino, C. Redmond et al., "Lumpectomy compared with lumpectomy and radiation therapy for the treatment of intraductal breast cancer," New England Journal of Medicine, vol. 328, no. 22, pp. 1581-1586, 1993.

[24] B. Fisher, S. Land, E. Mamounas, J. Dignam, E. R. Fisher, and N. Wolmark, "Prevention of invasive breast cancer in women with ductal carcinoma in situ: an update of the National Surgical Adjuvant Breast and Bowel Project experience," Seminars in Oncology, vol. 28, no. 4, pp. 400-418, 2001.

[25] I. L. Wapnir, J. J. Dignam, B. Fisher et al., "Long-term outcomes of invasive ipsilateral breast tumor recurrences after lumpectomy in NSABP B-17 and B-24 randomized clinical trials for DCIS," Journal of the National Cancer Institute, vol. 103, no. 6, pp. 478-488, 2011.

[26] J. Houghton, "Radiotherapy and tamoxifen in women with completely excised ductal carcinoma in situ of the breast in the UK, Australia, and New Zealand: randomised controlled trial," Lancet, vol. 362, no. 9378, pp. 95-102, 2003.

[27] J. Cuzick, I. Sestak, S. E. Pinder et al., "Effect of tamoxifen and radiotherapy in women with locally excised ductal carcinoma in situ: long-term results from the UK/ANZ DCIS trial," The Lancet Oncology, vol. 12, no. 1, pp. 21-29, 2011.

[28] S. Emdin, B. Granstrand, A. Ringberg et al., "SweDCIS: radiotherapy after sector resection for ductal carcinoma in situ of the breast. Results of a randomised trial in a population offered mammography screening," Acta Oncologica, vol. 45, no. 5, pp. 536-543, 2006.

[29] L. Holmberg, H. Garmo, B. Granstrand et al., "Absolute risk reductions for local recurrence after postoperative radiotherapy after sector resection for ductal carcinoma in situ of the breast," Journal of Clinical Oncology, vol. 26, no. 8, pp. 12471252, 2008.

[30] A. Omlin, M. Amichetti, D. Azria et al., "Boost radiotherapy in young women with ductal carcinoma in situ: a multicentre, retrospective study of the Rare Cancer Network," Lancet Oncology, vol. 7, no. 8, pp. 652-656, 2006.

[31] P. Wong, C. Lambert, R. V. Agnihotram, M. David, M. Duclos, and C. R. Freeman, "Ductal carcinoma in situ-the influence of the radiotherapy boost on local control," International Journal of Radiation Oncology Biology Physics, vol. 82, no. 2, pp. e153-e158, 2012.

[32] M. A. Ben-David, D. E. Sturtz, K. A. Griffith et al., "Long-term results of conservative surgery and radiotherapy for ductal carcinoma in situ using lung density correction: the University of Michigan experience," Breast Journal, vol. 13, no. 4, pp. 392400, 2007.

[33] L. J. Solin, A. Fourquet, F. A. Vicini et al., "Mammographically detected ductal carcinoma in situ of the breast treated with breast-conserving surgery and definitive breast irradiation: long-term outcome and prognostic significance of patient age and margin status," International Journal of Radiation Oncology Biology Physics, vol. 50, no. 4, pp. 991-1002, 2001.

[34] L. J. Solin, A. Fourquet, F. A. Vicini et al., "Salvage treatment for local or local-regional recurrence after initial breast conservation treatment with radiation for ductal carcinoma in situ," European Journal of Cancer, vol. 41, no. 12, pp. 17151723, 2005.

[35] E. S. Wai, M. L. Lesperance, C. S. Alexander et al., "Effect of radiotherapy boost and hypofractionation on outcomes in 
ductal carcinoma in situ," Cancer, vol. 117, no. 1, pp. 54-62, 2011.

[36] A. Monteau, B. Sigal-Zafrani, Y. M. Kirova et al., "Ductal carcinoma in situ of the breast with close or focally involved margins following breast-conserving surgery: treatment with reexcision or radiotherapy with increased dosage," International Journal of Radiation Oncology Biology Physics, vol. 75, no. 4, pp. 1021-1028, 2009.

[37] D. Azria, H. Auvray, I. Barillot et al., "Ductal carcinoma in situ: role of the boost," Cancer/Radiotherapie, vol. 12, no. 6-7, pp. 571-576, 2008. 


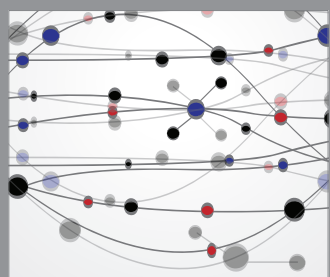

The Scientific World Journal
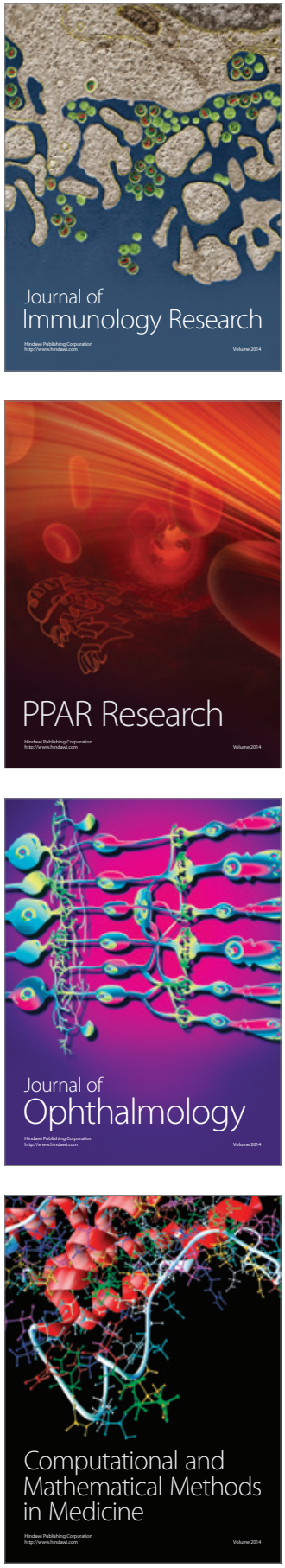

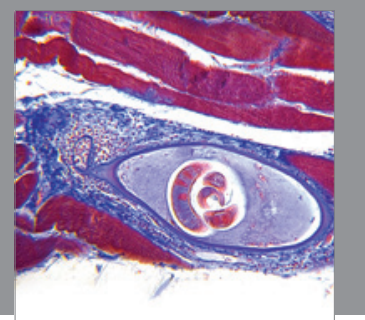

Gastroenterology

Research and Practice
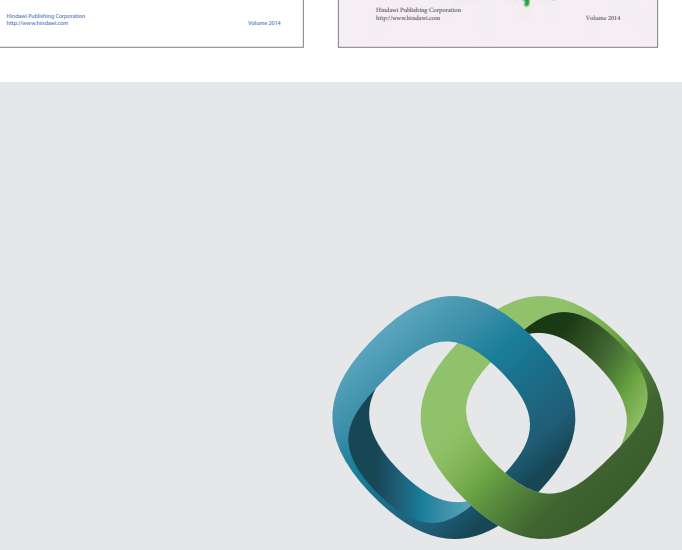

\section{Hindawi}

Submit your manuscripts at

http://www.hindawi.com
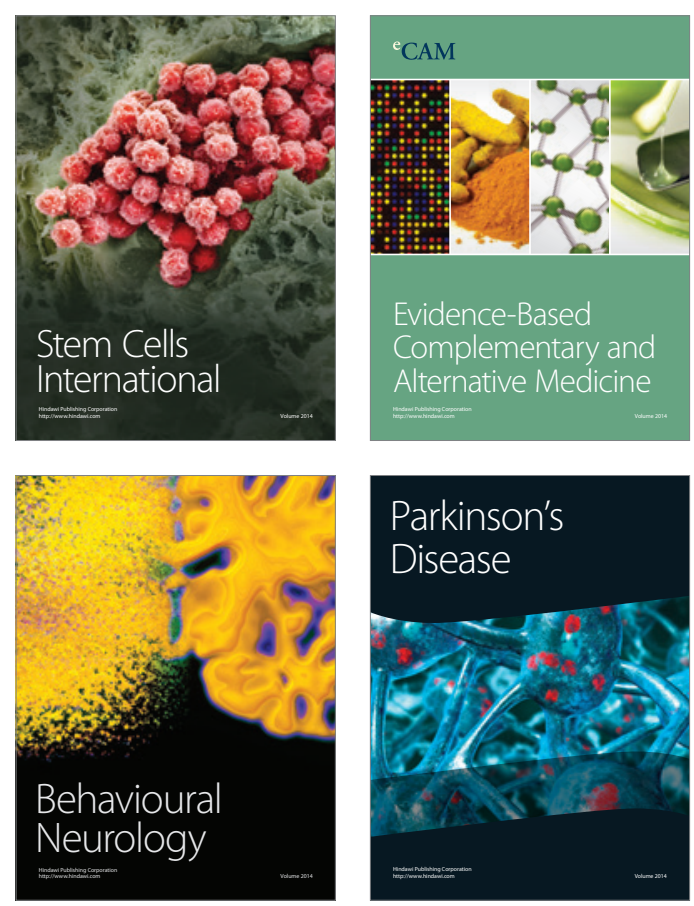

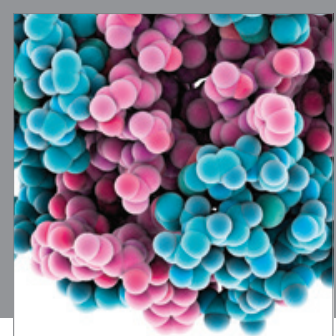

Journal of
Diabetes Research

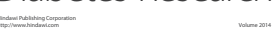

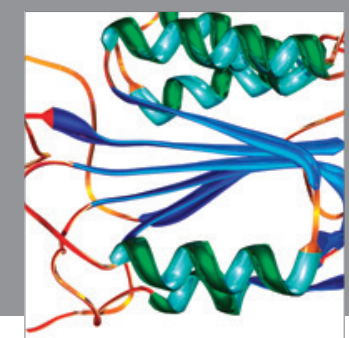

Disease Markers
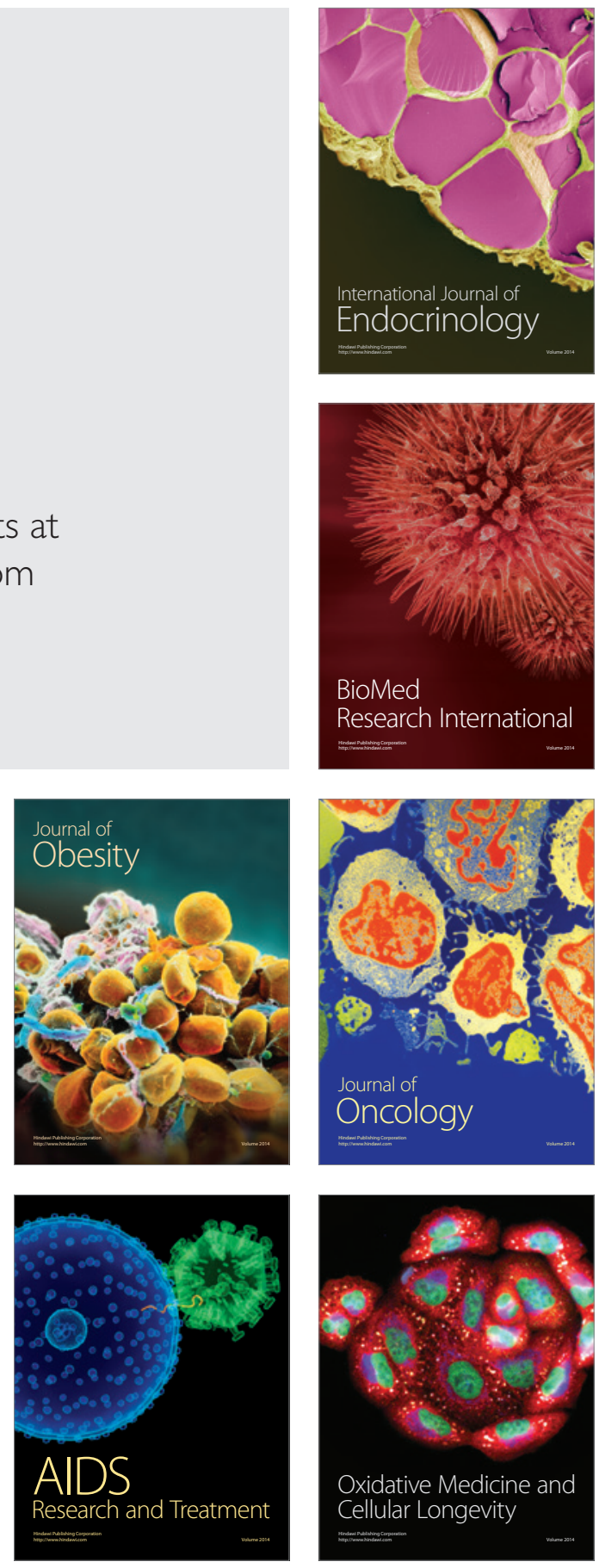